\title{
Using clinical indicators in emergency medicine: documenting performance improvements to justify increased resource allocation
}

\author{
Ian R Rogers, Lynne Evans, George A Jelinek, Ian Jacobs, Cathie Inkpen, David Mountain
}

\begin{abstract}
Objectives-To demonstrate how emergency department triage scale and thrombolysis indicator data can be used to document the impact of a substantial increase in resource allocation.

Methods-Descriptive study in an emergency department of an adult tertiary hospital in Perth, Australia during similar periods of the year both before and after a substantial increase in emergency department staff, equipment, and system resources. The study group comprised a total of 11048 emergency department attendances and all cases of emergency department initiated thrombolysis or acute angioplasty. Outcome was measured using numbers seen and percentage seen within indicator threshold time together with admission rates in each of the five triage categories as well as by using time from presentation to initiation of reperfusion treatment in acute myocardial infarction. Results-The proportion of patients seen within the prescribed indicator time increased by $16.4 \%$ (95\% confidence interval $14.4 \%$ to $18.2 \%)$. The increase was most pronounced in triage category $2(32.7 \%)$. Median time to thrombolysis fell by 30 minutes to 37 minutes $(p=0.0002)$.

Conclusions-Use of the Australasian national triage scale and time to thrombolysis clinical indicator data allows a quantitative assessment of the impact of increased emergency department resource allocation.

$(\Im$ Accid Emerg Med 1999;16:319-321)
\end{abstract}

Keywords: clinical indicators, triage, thrombolysis

For managers, one of the challenges of modern health administration is to provide objective, quantitative evidence that increased resource allocation translates into improved care for patients. Health care organisations have a responsibility to provide quality care for their patients and carers and to be accountable for the outcomes of care and services. ${ }^{1}$

In Australia, the Australian Council on Healthcare Standards is a major advocate of quality in health care. The Evaluation and Quality Improvement Program (EQuIP) has been developed in association with health care providers to assist organisations to deliver high quality health care. EQuIP establishes accountability mechanisms that assist organisations to demonstrate outcomes and focus on continuous improvement. It is a management tool that provides a framework for establishing a culture of continuous improvement. The Sir Charles Gairdner Hospital uses the EQuIP principles and framework for documentation and evidence of improved outcomes. Through EQuIP the hospital assesses its own performance against the principles of leadership, best practice, improving performance, and outcomes. An external survey confirms the self assessment through practical evidence of meeting all standards and documenting outcomes.

Performance and achievements can be monitored by the use of clinical indicators. These are defined as "measures of the management and outcome of care". ${ }^{1}$ Clinical indicators are flags that can alert to opportunities for improvement in patient care. They lend objectivity and interest to quality activities by allowing for comparison of performance against thresholds and national aggregate data. Their use may also identify areas for further quality activities, generate ideas for new studies, and lead to the development of organisation specific indicators. ${ }^{1}$

During 1996 the Sir Charles Gairdner Hospital identified emergency medicine as a key strategic direction in response to a failure to meet widely accepted performance targets. The aim of this study was to show how indicator data were used to show the objective improvements in patient care that followed a substantial increase in resource allocation to the emergency department.

\section{Methods}

DATA COLLECTION

The Australasian national triage scale allocates a medical priority to all patients on arrival at the emergency department on the basis of a brief and directed nursing assessment. In response to the proposition, "this patient should wait no longer than ...x ... minutes for medical care", the triage nurse places the 
Table 1 Attendance, waiting times, and proportion admitted by triage category before and after intervention

\begin{tabular}{|c|c|c|c|c|c|c|}
\hline & \multicolumn{5}{|c|}{ Triage category } & \multirow[b]{2}{*}{ All cases } \\
\hline & 1 & 2 & 3 & 4 & 5 & \\
\hline \multicolumn{7}{|l|}{ April to May 1997} \\
\hline Total attendance (No) & 90 & 645 & 2266 & 2201 & 317 & 5519 \\
\hline (\%) & 1.6 & 11.7 & 41.1 & 39.9 & 5.7 & 100.0 \\
\hline Seen within indicator time (\%) & 100.0 & 37.7 & 48.1 & 60.6 & 84.9 & 54.7 \\
\hline Admitted (\%) & 85.6 & 62.8 & 49.7 & 29.6 & 13.6 & 41.7 \\
\hline \multicolumn{7}{|l|}{ April to May 1998} \\
\hline Total attendance (No) & 88 & 1019 & 2284 & 1937 & 201 & 5529 \\
\hline (\%) & 1.6 & 18.4 & 41.3 & 35.0 & 3.7 & 100.0 \\
\hline Seen within indicator time (\%) & 100.0 & 70.4 & 68.5 & 73.4 & 87.1 & 71.7 \\
\hline Admitted (\%) & 96.6 & 64.0 & 51.1 & 28.3 & 6.0 & 44.6 \\
\hline
\end{tabular}

patient into one of five triage categories. The aim is for patients in category 1 (resuscitation) to be seen immediately, category 2 (emergency) within 10 minutes, category 3 (urgent) within 30 minutes, category 4 (semiurgent) within 60 minutes, and category 5 (nonurgent) within 120 minutes. ${ }^{2}$ Arrival times, triage codes, and time of first medical assessment were collected prospectively onto a computer data base for all emergency department attendances. The admission rate is considered the total of admissions, transfers, and deaths in the department divided by total attendances.

Reperfusion treatment data (on emergency department initiated thrombolysis and primary angioplasty patients) is maintained on a separate database. This records time of arrival, time to the start of reperfusion treatment, and a number of intermediate time points. These include the time electrocardiography (ECG) is first done, time of decision to reperfuse, and drug administration times. Although not included in this review, these intermediate data are also used for quality improvement activities.

The figures presented for the triage data are for the period April to May 1997 (before intervention) and April to May 1998 (after intervention). Those for the thrombolysis data compare January to April 1997 (before intervention) to January to April 1998 (after intervention). These periods were selected as lying clearly on either side of interventions expected to impact on the observed outcomes. Our hospital is one of the two major tertiary referral hospitals in the region and sees only adult patients. Emergency department attendances are of the order of 35000 per year with a $45 \%$ admission rate.

INTERVENTIONS

Before intervention the emergency department had been run by locum consultant staff with many from other specialties including general medicine and intensive care. Initial permanent appointments were made of Professor and Chairman of Emergency Medicine, Director of Emergency Medicine, and Director of Trauma/Retrieval. Another six consultant appointments were then made, producing a total of nine consultant staff, all with specialist emergency medicine qualifications. Total registrar and resident numbers were left unchanged at 25 whole time equivalents but several resident medical officer posts were converted to registrar posts, leading to all junior medical staff being supervised directly by a registrar.

The new appointment of a clinical staff educator was made to oversee nurse education and clinical practice. A year long emergency nursing course was started in conjunction with a local university. Students participating in this course work under direct supervision within the emergency department. Nursing numbers remained unchanged at 47 whole time equivalents.

Clerical functions in the emergency department were boosted by the creation of a liaison officer role. This position, staffed for 128 out of a 168 hour week, had specific responsibilities relating to incoming and outgoing telephone calls, maintenance of a patient register, and indicator data collection.

A minor refurbishment was undertaken to maximise the utility of clinical space available in the emergency department. Initiatives included creation of six extra cubicles, redesigned data collection and information systems, and purchase of upgraded equipment such as more ECG machines to support new clinical initiatives.

Emergency department controlled actual expenditure increased by $\$$ A 1.95 million or $41 \%$ from the before to after intervention periods. The true total cost of all the measures is more difficult to define given the impact of changing emergency department function on other departments such as pathology, radiology, the intensive care unit, and the general wards.

A wide range of clinical guidelines and clinical pathways was developed using a consensus approach with consultation both within the emergency department and with services outside the department. Examples included a coordinated trauma team response, youth suicide prevention, an asthma clinical pathway, and the start of emergency department initiated thrombolysis. The aim of these interventions was to improve both the quality and timeliness of patient care.

Statistical analysis was undertaken using the SPSS software (version 8.0). Estimates for each measure were calculated using univariate analysis with the corresponding $95 \%$ confidence interval $(\mathrm{CI})$ constructed. The $\chi^{2}$ test was used to determine differences between triage categories. Differences between time to thrombolysis were determined using the Mann-Whitney test. Significance was set at 0.05 .

\section{Results}

There were 5519 and 5529 patients seen in the emergency department during the 1997 and 1998 data collection periods respectively. The mean waiting time for the period before intervention in 1997 was 49 minutes. This had fallen by 20 minutes in the period after intervention in 1998. Overall the proportion of patients seen within the prescribed indicator time increased over the study period by $16.4 \%$ (95\% CI $14.4 \%$ to $18.2 \%)$. This increase occurred in triage categories $2-5$ but was most striking in triage category 2 where there was a $32.7 \%$ improvement $\left(\chi^{2}=127.0, \mathrm{df}=4\right.$, $\mathrm{p}=<0.0001)$. All patients in triage category 1 
were seen immediately upon arrival. These results are summarised in table 1 .

There was a small increase of $2.9 \%(95 \% \mathrm{CI}$ $1.1 \%$ to $4.7 \%$ ) in the overall proportion of patients admitted to hospital from the emergency department between the before and after intervention periods. As presented in table 1, small increases were observed in the higher acuity triage categories 1-3 with fewer patients being admitted from triage categories 4 and 5 .

As with other clinical indicators measured the time taken to initiate thrombolysis significantly improved over the study period. In 1997 ( $n=12$ ) the median "door to needle" time was 67 minutes (95\% CI 53 to $107 \mathrm{~min}$ ) and by 1998 ( $\mathrm{n}=37$ ) this had fallen to 37 minutes (95\% CI 33 to $45 \mathrm{~min}$ ). This improvement was highly significant (Mann-Whitney $=3.475$, $\mathrm{p}=0.0002$ ).

\section{Discussion}

The Australasian national triage scale has progressed substantially from its beginnings in the 1970 s. $^{34}$ Initially developed as a way of prioritising medical care to emergency department patients, it is now used a clinical indicator, bench marking tool, and funding mechanism. ${ }^{56}$ Its great strengths lie in its simplicity, reproducibility, and applicability in a wide variety of environments. ${ }^{7-10}$ The simple inputs required to obtain these indicator data are collected in real time and measure end points that relate directly to the quality of patient care and are meaningful to the clinical staff involved.

Our data demonstrate substantial variations in workload and performance between the before and after intervention periods. The most striking difference in total numbers seen in the two periods is in triage category 2 . This is a highly resource intensive category and typically includes patients with problems such as ischaemic chest pain. This increase probably explains the increased number of patients with acute myocardial infarction undergoing reperfusion treatments. The shortened mean waiting time in category 2 and the shortened median time to thrombolysis reflect improved quality of care provided to this group. We can only speculate on the reasons for our number of category 2 patients increasing but believe it is likely to reflect an increasing confidence in the department from referring local doctors and ambulance officers. These changes are probably due to a redistribution of cases previously taken to other hospitals in the region. There is no reason to believe that the incidence of serious illness in our community could change so suddenly from year to year.

Importantly, a useful clinical indicator should not be open to manipulation and gaming. The admission rate noted within each national triage scale category provides both an internal and external check on the accuracy of the triage process. " "Up-triaging" to inflate high acuity workload inevitably leads to a decline in the admission rate in the higher triage categories. Paradoxically "up-triaging" tends to produce a worsening of the percentage seen within time rate and so the two forces tend to balance out.
Indicator admission rates in each triage category are widely published, ${ }^{910}$ and all our rates fall within or close to these guidelines. Notably in our study, the admission rates were only slightly altered in the key triage categories 2,3 , and 4 confirming that our application of the national triage scale did not vary significantly between the two study periods.

Time to thrombolysis or other reperfusion treatment is a valuable emergency department clinical indicator. As well as being a measure of the performance of the system as a whole (including clerical, nursing, and medical inputs) it is clear that a shortened time to thrombolysis saves patient lives and improves quality of life. ${ }^{11}$ The changes we introduced by moving to an emergency department initiated thrombolysis system produced results on a par with the best reported in our region to date..$^{12}$ Again, the simplicity of the data and its clinical relevance are key features of time to thrombolysis as a clinical indicator.

Which of the many interventions produced the most substantial improvements is difficult to judge. We believe that they must be seen as a package. Our focus was staffing the department with senior and experienced clinicians committed to providing high quality care. In summary, we have shown that by collection of simple and reliable triage scale and time to thrombolysis data we can demonstrate substantial improvements in the quality of care provided to emergency department patients. This was essential to justify the increased resource allocation provided to our department. We commend the use of these clinical indicators to other organisations as a measure of the quality of care they provide to their patients.

Conflict of interest: none.

Funding: none.

1 Australian Council on Healthcare Standards. The EQuIP guide: standards and guidelines for the ACHS evaluation and quality improvement program. Sydney: Australian Council on Healthcare Standards, 1996.

2 Australasian College for Emergency Medicine. Policy document. National triage scale. Emergency Medicine 1994;6: $145-6$.

3 Brentnall EW. A history of triage in civilian hospitals in Australia. Emergency Medicine 1997;9:50-4.

4 Pink N. Triage in the accident and emergency department. Australian Nurses fournal 1977;6:35-6.

5 Erwich-Nijhout AE, Bond MJ, Phillips DG, et al. The identification of costs associated with emergency departmen attendances. Emergency Medicine 1997;9:301-5.

6 Bond MJ, Erwich-Nijhout AE, Phillips DG, et al. Urgency, disposition and age groups: a case mix model fo emergency medicine. Emergency Medicine 1998;10:103-10

7 Jelinek GA, Little M. Inter rater reliability of the national triage scale over 11,500 simulated occassions of triage. Emergency Medicine 1996;8:226-30.

8 Hollis G, Sprivulis P. Reliability of the national triage scale with changes in emergency department activity level. Emergency Medicine 1996;8:231-4.

9 Browne GJ, Gaudry PL, Lam L. A triage observation scale improves the reliability of the national triage scale. Emproven the reliability of the

10 Standen P, Dilley SJ. A review of triage nursing practice and experience in Victorian public hospitals. Emergency Medicine 1997;9:301-5.

11 Rawles J. Magnitude of benefit from earlier thrombolytic treatment in acute myocardial infarction: new evidence from the Grampian region early anistreplase tria (GREAT). BMf 1996;312:212-15.

12 Jacobs IG, Fatovich DM. The use of thrombolytic therapy in patients presenting to a peripheral metropolitan emergency department with acute myocardial infarction. Aust N Z F Med 1996;26:539-42.

13 Hitchcock T, Kidd HM, Taylor R. Time delay in the treatment of acute myocardial infarction (AMI): a comparison of primary percutaneous transluminal coronary angioplasty (PTCA) with thrombolysis. Aust $N Z \mathscr{Z}$ Med 1997;27:531-7. 